Research paper

\title{
General Practitioners' use of and attitudes to acupuncture in relation to the UK's National Institute for Health and Care Excellence (NICE) clinical guidelines-A pilot study
}

\author{
Iga Czarnawska-Iliev*, Nicola Robinson, Prof. ${ }^{1}$ \\ School of Health and Social Care, London South Bank University, United Kingdom
}

\section{A R T I C L E IN F O}

\section{Article history:}

Received 24 May 2016

Received in revised form 17 July 2016

Accepted 17 July 2016

Available online $\mathrm{xxx}$

\section{Keywords:}

Clinical guidelines

Acupuncture

Primary care

Integration

NICE

Attitudes

General practitioners

\begin{abstract}
A B S T R A C T
Introduction: Until April 2016, acupuncture in the UK was recommended by the National Institute for Health and Care Excellence (NICE) as a potential treatment modality for three conditions, but use of this guidance in primary care is unknown. The aim of this study was to update the mapping of acupuncture on NICE clinical guidelines and to explore general practitioners' (GPs') awareness of those guidelines, as well as their views on and referral to acupuncture. It also examined the feasibility of research through electronic questionnaires administered to GPs.

Methods: Initially, a literature search was conducted of NICE guidelines mentioning acupuncture (up to July 2015). Subsequently, a random sample of 57 GPs in North London was asked to complete an electronic survey.

Results: Literature search identified one new "do not offer" recommendation (CG171: Urinary Incontinence). Four guidelines discussed acupuncture, concluding evidence was insufficient. The survey yielded 19 responses from 34 potential respondents. Patient demand appeared widespread but small; several GPs received enquiries but provided no access. The most common reason for enquiry was pain management. Importance assigned to guidance and awareness of guidance other than for pain varied significantly: GPs' decision to offer access did not correlate with guideline awareness. GPs often expected recommendations where there were none. GPs professing least trust in guidance appeared more likely to offer acupuncture access.

Conclusion: NICE guidelines appeared not to reflect acupuncture provision in primary care. Electronic questionnaires are a feasible research method in primary care, although obtaining up-to-date contact details poses a challenge.
\end{abstract}

(c) 2016 Published by Elsevier GmbH.

\section{Introduction}

In England, clinical guidance is developed by the National Institute for Health and Care Excellence (NICE), ${ }^{2}$ a non-

Abbreviations: CAM, Complementary and Alternative Medicine; CCG, Clinical Commissioning Group; GP, General Practitioner; NHS, National Health Service; NICE, National Institute for Health and Care Excellence.

* Corresponding author at: 18 Aintree Rd, Royston, SG8 9JE, United Kingdom.

E-mail addresses: iga.czar@gmail.com (I. Czarnawska-Iliev),

nicky.robinson@lsbu.ac.uk (N. Robinson).

1 Traditional Chinese Medicine (TCM) and Integrated Health, School of Health and Social Care, London South Bank University, 103 Borough Road, London SE1 OAA United Kingdom.

2 The naming of this institution occasionally presents some confusion. Between 1999 when it was established and 2005, NICE was known as the National Institute for Clinical Excellence. In 2005, it merged with the Health Development Agency, changing its name to the current one. departmental public body. NICE guidelines are developed by Guidance Development Groups, who determine review questions relating to the considered condition or intervention and search for evidence that best answers them.

In the most recent review of NICE clinical guidelines in the UK [1], acupuncture was reported to be the most frequently mentioned Complementary and Alternative Medicine (CAM), discussed in 35 guidelines, and, at that time, recommended as one of the treatment options for three conditions. These were: low back pain in Clinical Guideline 88: Low back pain: early management of persistent non-specific low back pain, 2009 (CG88), and for headaches and migraines in Clinical Guideline 150: Headaches: diagnosis and management of headaches in young people and adults, 2011 (CG150). At the time of writing, those remained the only guidelines recommending acupuncture as a potential treatment modality, while Clinical Guideline 62: Pregnancy Care (CG62), recommended discussing wrist acupressure for pregnancy nausea, 
while informing women to minimise their use of CAM in general. However a revised version of CG88, currently under consultation until May 6th, 2016, does not include acupuncture as a recommendation. Most guidelines which discuss acupuncture conclude it cannot be recommended based on weak or insufficient evidence (e.g. CG70: Labour induction; CG111: Nocturnal Enuresis; CG 120: Psychosis with coexisting substance misuse).

At the same time, systematic reviews point to a substantial body of evidence for acupuncture use for other conditions: for instance, neck pain [2,3], obstetric conditions [4], and chronic pain conditions, including shoulder and osteoarthritis [5]. Hence, international clinical guidelines sometimes include acupuncture recommendations which are not endorsed by NICE, such as the 2007 Guidelines of the American College of Chest Physicians for lung cancer, or the French Haute Autorité de Santé guidance for Nausea and Vomiting of Pregnancy [6].

Additionally, the relationship between guidelines and clinical practice is often questioned. Reports suggest that general practitioners may be "critical" of such guidance [7] and "not implementing the guidelines they disagree with" [8].

To the best of the authors' knowledge, GPs' awareness and implementation specifically of acupuncture guidelines has not yet been studied in the UK. Also, no studies of GPs' attitudes to guidelines have been conducted since 2009, when acupuncture was first mentioned by NICE as a potential recommendation in CG88.

A better understanding of the inter-relation between GPs, acupuncture and guidelines can inform ways to enable patients to benefit from the choice of more treatment options. Should patients require acupuncture for a condition not supported by NICE, they have to cover the cost, which may constitute a barrier to treatment. In 2009, of the 4 million reported annual acupuncture treatments, about one-third were provided within the NHS, the remaining being private [9]. In order to enable equal access to beneficial treatment modalities, the question of whether official bodies such as NICE develop guidelines which match the realities of patientdoctor interaction becomes ever more pertinent.

Furthermore, some researchers specify advantages of CAM integration for the NHS, such as lower long-term costs of care [10]. Additionally, examining GPs' attitudes to NICE guidelines for acupuncture can help illuminate whether the time and budget of national organisations is spent developing guidelines that serve the intended public, or "fall on stony ground", as Rashidian et al. [7] suggested of guidelines. However, as GPs are facing increasing time pressures, research involving this group is notoriously difficult [11,12].

In the view of the above, there appears to be a gap in research pertaining to the use of NICE guidelines relating to acupuncture in primary care. Phase one of this study aimed at updating the mapping of acupuncture within NICE guidelines. This information was used in phase two, which piloted an electronic questionnaire to assess the feasibility of questionnaire-based research methods in general practice. The research objectives of phase two were to explore, firstly, acupuncture usage in primary care, secondly, GPs' attitudes to this treatment modality and thirdly, their awareness and attitude to official NICE guidelines for acupuncture.

\section{Methods}

\subsection{Study design}

There were two parts to this study. The first part consisted of generating an updated review of NICE guidelines on acupuncture, by tracing which NICE guidelines provide a recommendation relating to acupuncture and the specific recommendations for each guideline. The second part was a pilot survey of a random sample of
General Practitioners in a multi-ethnic London Borough, (Brent) using an electronic questionnaire.

\subsection{Part 1: reviewing NICE guidance on acupuncture}

It was intended for this phase to update the 2014 review by Lorenc et al. [1], but focusing only on acupuncture. The initial search covered a time period which overlapped with Lorenc's review, in order to be cross-checked with that review to assess the accuracy of the search method. The NICE website was searched for the keyword "acupuncture" within clinical guidance. This yielded 38 hits; leading to 13 clinical guidelines. Cross-checking revealed that there were four guidelines discussed by Lorenc et al. as relevant to acupuncture (CG120; CG143; CG52 and CG112) which had not been identified by our search. As a result; the initial search process was proved inadequate and was modified. The full text of all guidelines published between July 2013 and July 2015 was searched for the keyword "acup*"; allowing identification of both acupuncture and acupressure.

The updated list of guidelines pertaining to acupuncture was then used in Part Two of the study, to assess correct recognition of guidelines by questionnaire respondents.

\subsection{Part 2: electronic questionnaire survey of general practitioners}

\subsubsection{Data collection}

A random sample of 57 General Practitioners from the London Borough of Brent were sent an email invitation to complete a questionnaire survey, hosted on SurveyMonkey (www.surveymonkey.com). The biggest challenge of the study was to obtain GP contacts and secure a sufficient response rate, hence the study was conducted through the Applied Research Unit in Brent CCG, who also handled the randomisation process. Participant inclusion criteria were:

- currently registered as a General Practitioner,

- working in the London Borough of Brent, irrespective of place of residence.

Required sample size was calculated based on Yamane's simplified formula [13]:

Population/ $\left(1+\right.$ population $\times$ sampling error $\left.{ }^{\wedge} 2\right)$

Consequently, the study aimed to collect 23 responses, in order to analyse results to a margin of error of 0.2 . An assumption about the expected response rate was made on the basis of studies into GP questionnaire responsiveness, which is notoriously low $[7,11,12]$. Taking into account that the collaboration with Brent's CCG raised the profile of the research, we assumed a response rate of around 30\%-35\% for this study, leading to an initial sample of 57. The initial mailing on the 26th of June 2015 was followed by two reminders, sent on the 21 st of July and the 5th of August 2015, with the view to increasing the response rate since there was a high probability that GPs would be on annual leave at this time of year.

\subsubsection{Questionnaire design and administration}

The questionnaire was electronic, largely quantitative with a qualitative component, and hosted on Surveymonkey.com (Appendix A). A questionnaire approach was chosen over interviews as less demanding on the time of the respondents.

The questionnaire was designed specifically for this pilot study, based on the guidance offered in Oppenheim [14] and Foddy [15]. There was consultation with the research unit in Brent CCG prior to use. There were four parts: (1) demand for and provision of acupuncture, (2) attitude to acupuncture, (3) NICE guideline 
recognition and attitudes, and (4) a demographic section. The three first parts comprised 12 items and an optional open comment box, the demographic section had another 4 items. Most questions had a closed format, as this was thought of as timesaving and thus preferable for the respondent. The answer choices were based on interviews of GPs [16,17].

Since attitudes are seldom linear and measuring them is challenging, the questionnaire asked respondents to gauge both the risk and benefit of acupuncture, as well as whether they would like NICE to consider it for more conditions, and - to estimate trust in guidance - how likely they would be to follow a NICE recommendation for a condition they suffered from. Respondents also had the option to express their opinions directly in the open comment box.

Assessing awareness of NICE recommendations was based on expecting a positive answer only where a recommendation was present. All other options (an active recommendation of 'do not offer' or "do not use", a lack of recommendation, usually phrased in the guideline as "cannot recommend" and a lack of mention within the guideline) were collapsed into a negative answer.

\subsubsection{Sample characteristics}

Brent is a North-West London borough, characterised by great social contrasts and ethnic heterogeneity. It has the lowest proportion of residents born in the UK (45\%), however, most are long-term immigrants, who arrived in England as children [18]. Therefore, country of education rather than ethnicity was measured in the questionnaire. Contrasts in terms of wealth and education have implications for healthcare: the gap in life expectancy for males from the most and least affluent parts of the borough is almost 10 years [19].

As of April 2015 there were 67 GP surgeries in Brent, jointly caring for over 360,000 patients [20]. The number of GPs was assessed by checking individual surgeries' websites (June-July 2015) and contacting two practices who did not provide adequate information. There were 236 practising GPs and 55\% were female.

\subsubsection{Data analysis}

The approach to the data was chiefly exploratory, since the sample size was too small to render meaningful results in statistical tests. Descriptive statistics, prepared using Microsoft Excel and Office Libre Calc, were used to summarise and compare across different variables. Additionally, SPSS and R were used to compute Cronbach Q Pearson's Chi Squared test and binomial distribution where appropriate.

When measuring risk, for data analysis, the 10-point scale was divided 30\%-70\%, to compare the incidence of "Low Risk" versus "Higher Risk" responses. This rather than a symmetrical division was chosen as more appropriate when discussing of a possible medical intervention, where safety is paramount.

Also, respondents were subdivided based on their attitude to NICE guidance into "Low trust", "High Trust" and "Neutral" groups, and the extreme groups' profiles were compared with regards to correct guideline recognition, acupuncture access provision (use or referral) and acupuncture attitude.

Open ended comments were analysed qualitatively using the Framework analysis method [21]. As the link to the original respondent was preserved, it was possible to compare emergent themes with quantitative data where appropriate. All comments were analysed as a single data set and coded thematically by one researcher; this was considered sufficient given a modest research sample.

\subsection{Ethical considerations}

The study was approved by LSBU's Research Ethics Committee as a Masters research project, while NHS Ethics approval was not needed. Participant consent was implied by clicking on the link to the questionnaire, which respondents could quit at any time. Confidentiality was assured by sending the survey over an SSL encrypted connection. Efforts to minimise the time commitment of the already time-pressured GPs included careful editing of the questionnaire, limiting the number of reminders and precluding non-respondent sampling.

\section{Results}

\subsection{Part 1: NICE guidance review}

There were 36 guidelines published between July 2013 and July 2015. 10 of these were updates of previous guidelines. Six, including 4 updates, discussed acupuncture within available evidence. 4 of these concluded it was lacking and did not mention acupuncture in the guidance recommendations section. In the questionnaire, those guidelines mapped onto "Cancer and associated conditions", "Neurological" and "Psychiatric illness". The remaining 2 guidelines made a "do not offer" recommendation. Of those, CG177: Osteoarthritis: care and management in adults, was an update of CG59, while CG171: Urinary Incontinence: The management of bedwetting in children and young people, provided a new "do not offer" recommendation. Additionally, CG190: Intrapartum care, updating CG55, discussed evidence for acupressure in labour, also concluding it was insufficient and recommending: "do not offer, but do not prevent women from using it".

Incidentally, the first part of the search, which was crosschecked with the previous review of CAM in clinical guidance [1], lead to what appeared a different conclusion in two cases. Guidelines CG111: Nocturnal enuresis, and CG115: Alcohol Misuse, discuss acupuncture in the section on available evidence, making research recommendations, but do not mention it in guidance recommendations. In Lorenc et al., those guidelines are duly listed under "Mention CAM, but do not provide a recommendation, usually due to lack of evidence", and also annotated with "cannot recommend acupuncture". This conclusion is an inference by the researchers: implied by the guideline discussing acupuncture research and not making a recommendation for or against its use. However, we looked for verbatim statements of "recommend", "cannot recommend" or "do not use", and the words "cannot recommend" were only used in this paper if employed verbatim in a NICE guidance. Such differences in interpretation may also influence GPs' understanding of these guidelines, therefore, the survey question on guidance recognition was formulated so that any lack of positive recommendation required a negative answer.

Details of the search results are summarised in Tables 1 and 2.

\subsection{Part 2: survey of general practitioners}

\subsubsection{Participation rates and respondent characteristics}

Several addresses bounced (Fig. 1), eventually yielding a number of 34 potential respondents. A total of 19 questionnaire responses were collected (56\% response rate), of which 3 were incomplete - two had missing demographic data, and one over a half of responses. All responses were included in the data set, with missing data reported where appropriate. Eight participants agreed to receive further contact from the researcher and four provided their email addresses. 
Table 1

NICE guidelines discussing acupuncture. Original NICE wording kept to reflect the strength level of recommendation.

\begin{tabular}{|c|c|c|}
\hline $\begin{array}{l}\text { Guideline (date } \\
\text { published) }\end{array}$ & Condition & Conclusion \\
\hline CG52 (July 2007) & Drug misuse & cannot recommend \\
\hline CG59 (Feb 2008) & Osteoarthritis & electro-acupuncture should not be used \\
\hline CG60 (Feb 2008) & Otitis media with effusion & not recommended \\
\hline CG61 (Feb 2008) & Irritable bowel syndrome & should not be encouraged \\
\hline $\begin{array}{l}\text { CG62 (March } \\
\text { 2008) }\end{array}$ & $\begin{array}{l}\text { Antenatal care: routine care for the } \\
\text { healthy pregnant woman }\end{array}$ & $\begin{array}{l}\text { recommends wrist acupressure for nausea; } \\
\text { discusses acupuncture, recommends informing women to minimise CAM usage. ("Pregnant women should } \\
\text { be informed that few complementary therapies have been established as being safe and effective during } \\
\text { pregnancy }[\ldots \text {. } . \text { they should be used as little as possible") }\end{array}$ \\
\hline CG70 (July 2008) & Induction of labour & inform women that "available evidence does not support acupuncture" for labour induction \\
\hline CG88 (May 2008) & Persistent, non-specific low back pain & recommends \\
\hline CG97 (May 2010) & Lower urinary tract infection in men & do not offer \\
\hline CG98 (May 2010) & $\begin{array}{l}\text { Neonatal jaundice - } \\
\text { hyperbilirubinaemia }\end{array}$ & do not use \\
\hline CG111 (Oct 2010) & Nocturnal enuresis & $\begin{array}{l}\text { research recommendation, no mention within the guidance recommendations } \\
\text { (In Lorenc et al. [1]: "cannot recommend") }\end{array}$ \\
\hline CG112 (Dec 2010) & Sedation in children and young adults & cannot recommend \\
\hline CG115 (Feb 2011) & Alcohol misuse & $\begin{array}{l}\text { research recommendation, no mention within the guidance recommendations } \\
\text { (In Lorenc, 2014: "cannot recommend") }\end{array}$ \\
\hline $\begin{array}{l}\text { CG120 (March } \\
\text { 2011) }\end{array}$ & $\begin{array}{l}\text { Psychosis with coexisting substance } \\
\text { misuse }\end{array}$ & cannot recommend \\
\hline CG 126 (July 2011) & Management of stable angina & do not offer \\
\hline CG143 (June 2012) & $\begin{array}{l}\text { Sickle Cell Aneamia acute painful } \\
\text { episode }\end{array}$ & $\begin{array}{l}\text { recommends discussion: "encourage the patient to use their own coping mechanisms (for example, } \\
\text { relaxation techniques, acupuncture, acupressure). }\end{array}$ \\
\hline CG150 (Sept. 2012) & Headaches & recommend: "Consider a course of up to 10 sessions" \\
\hline $\begin{array}{l}\text { CG171 (Sept 2013) } \\
\text { updates and } \\
\text { replaces CG40 }\end{array}$ & Urinary Incontinence & do not offer \\
\hline $\begin{array}{l}\text { CG177 (Feb 2014) } \\
\text { updates and } \\
\text { replaces CG59. }\end{array}$ & Osteoarthritis & do not offer \\
\hline $\begin{array}{l}\text { CG } 190(\text { Dec } 2014) \\
\text { updates and } \\
\text { replaces CG55. }\end{array}$ & Intrapartum Care & do not offer, but do not prevent women from using \\
\hline
\end{tabular}

Table 2

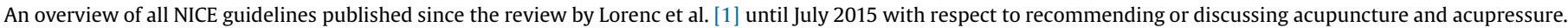

\begin{tabular}{|c|c|c|}
\hline $\begin{array}{l}\text { guideline (date } \\
\text { published) }\end{array}$ & condition & mentions acupuncture/acupressure? \\
\hline NG 14 (July 2015) & Melanoma: Assessment and Management & no mention \\
\hline NG 8 (June 2015) & $\begin{array}{l}\text { Anaemia management in people with } \\
\text { chronic kidney disease }\end{array}$ & no mention \\
\hline NG 12 (June 2015) & $\begin{array}{l}\text { Suspected Cancer: Recognition and } \\
\text { referral }\end{array}$ & no mention \\
\hline NG 9 (June 2015) & Bronchitis in Children & no mention \\
\hline NG 11 (May 2015) & $\begin{array}{l}\text { Challenging behaviour and learning } \\
\text { disabilities }\end{array}$ & no mention \\
\hline NG 10 (May 2015) & Violence and Aggression & no mention \\
\hline $\begin{array}{l}\text { NG } 5 \text { (March } \\
\text { 2015) }\end{array}$ & Medicines Optimisation & no mention (although discusses medication-based CAM) \\
\hline NG 2 (Feb 2015) & Bladder Cancer & no mention \\
\hline NG 3 (Feb 2015) & Diabetes in pregnancy & no mention \\
\hline NG 1 (Jan 2015) & $\begin{array}{l}\text { Gastro-oesophageal reflux disease in } \\
\text { children and young people }\end{array}$ & no mention \\
\hline CG 192 (Dec 2014) & Antenatal and postnatal mental health & $\begin{array}{l}\text { discusses evidence, but no recommendation. } \\
\text { "there was some evidence for depression-specific acupuncture and bright-light therapy. However the } \\
\text { GDG did not feel the evidence was strong enough to make any specific recommendations about physical } \\
\text { interventions." }\end{array}$ \\
\hline $\begin{array}{l}\text { CG } 190 \text { (Dec 2014) } \\
\text { updates and } \\
\text { replaces CG55. }\end{array}$ & Intrapartum Care & do not offer, but do not prevent women from using \\
\hline CG 191 (Dec 2014) & Pneumonia & no mention \\
\hline CG 189 (Nov 2014) & Obesity & no mention \\
\hline CG 187 (Oct 2014) & Acute Heart Failure & no mention \\
\hline CG 188 (Oct 2014) & Gallstone disease & no mention \\
\hline CG 186 (Oct 2014) & Multiple Sclerosis & $\begin{array}{l}\text { discusses evidence, but no recommendation: "Given the lack of clear clinical evidence and the } \\
\text { considerable cost to the NHS, the GDG felt further research was required into the use of acupuncture for } \\
\text { pain and spasticity in people with MS." }\end{array}$ \\
\hline $\begin{array}{l}\text { CG } 185 \text { (Sept } \\
\text { 2014) }\end{array}$ & Bipolar Disorder & $\begin{array}{l}\text { discusses evidence, but no recommendation: There was very low quality evidence that neither } \\
\text { acupuncture nor transcranial magnetic stimulation were associated with reductions in mania or } \\
\text { depression. }\end{array}$ \\
\hline $\begin{array}{l}\text { CG } 183 \text { (Sept } \\
\text { 2014) }\end{array}$ & Drug Allergy & no mention \\
\hline
\end{tabular}


Table 2 (Continued)

\begin{tabular}{|c|c|c|}
\hline $\begin{array}{l}\text { guideline (date } \\
\text { published) }\end{array}$ & condition & mentions acupuncture/acupressure? \\
\hline $\begin{array}{l}\text { CG } 184 \text { (Sept } \\
\text { 2014) } \\
\text { updates and } \\
\text { replaces CG17 }\end{array}$ & $\begin{array}{l}\text { Dispepsia and gastro-oesophageal reflux } \\
\text { disease }\end{array}$ & no mention \\
\hline $\begin{array}{l}\text { CG } 182 \text { (July 2014) } \\
\text { updates and } \\
\text { replaces CG } 73\end{array}$ & Chronic Kidney Disease & no mention \\
\hline CG 181 (July 2014) & $\begin{array}{l}\text { Lipid modification: cardiovascular risk } \\
\text { assessment }\end{array}$ & no mention \\
\hline $\begin{array}{l}\text { CG } 180 \text { (June } \\
\text { 2014) } \\
\text { updates and } \\
\text { replaces CG } 36\end{array}$ & Atrial fibrillation & no mention \\
\hline $\begin{array}{l}\text { CG } 176 \text { (April } \\
\text { 2014) }\end{array}$ & Pressure ulcers & no mention \\
\hline $\begin{array}{l}\text { CG } 177 \text { (Feb 2014) } \\
\text { Updates and } \\
\text { replaces CG57 }\end{array}$ & Osteoarthritis & "do not offer"; Discusses evidence, but concludes not cost-effective \\
\hline CG 178 (Feb 2014) & Psychosis and schizophrenia in adults & no mention \\
\hline $\begin{array}{l}\text { CG176 (Jan 2014) } \\
\text { updates and } \\
\text { replaces CG } 56\end{array}$ & Head Injury & no mention \\
\hline $\begin{array}{l}\text { CG175 (Jan 2014) } \\
\text { updates and } \\
\text { replaces CG } 58\end{array}$ & Prostate Cancer & $\begin{array}{l}\text { discusses evidence, concluding that: "men should be advised there is no good quality evidence for the } \\
\text { use of complementary therapies in the management of troublesome hot flushes", however, no mention } \\
\text { within recommendations. }\end{array}$ \\
\hline CG 174 (Dec 2013) & $\begin{array}{l}\text { Intravenous Fluid therapy in adults in } \\
\text { hospital }\end{array}$ & $\mathrm{N} / \mathrm{A}$ \\
\hline $\begin{array}{l}\text { CG } 172(\text { Nov 2013) } \\
\text { updates and } \\
\text { replaces CG48 }\end{array}$ & MI - secondary prevention & no mention \\
\hline $\begin{array}{l}\text { CG } 173(\text { Nov 2013) } \\
\text { updates and } \\
\text { replaces CG96 }\end{array}$ & $\begin{array}{l}\text { Neuropathic pain: pharmacological } \\
\text { management }\end{array}$ & (2) \\
\hline $\begin{array}{l}\text { CG } 171 \text { (Sept } \\
2013 \text { ) } \\
\text { updates and } \\
\text { replaces CG40 }\end{array}$ & Urinary Incontinence & $\begin{array}{l}\text { do not offer } \\
\text { discusses evidence, "Poor-quality evidence shows that acupuncture may reduce nocturia and both stress } \\
\text { and urgency incontinence in the short term". }\end{array}$ \\
\hline CG169 (Aug 2013) & Acute Kidney Injury & no mention \\
\hline CG170 (Aug 2013) & Autism & no mention \\
\hline CG167 (July 2013) & $\begin{array}{l}\text { Myocardial infarction with St-segment } \\
\text { elevation: acute management }\end{array}$ & no mention \\
\hline CG168 (July 2013) & Varicose veins in the legs & no mention \\
\hline
\end{tabular}

Demographic and education data was only available for 16 respondents. 5 out of 16 respondents were female, as compared to $55 \%$ of the general GP population in Brent. Three respondents received their medical training outside of Great Britain: one in the British Virgin Isles, one in Egypt and one in the USA. The most represented age range was $40-49$ years $(n=7)$, which is consistent with the national profile of GPs' age [22], however the 60+ age category seemed over-represented.

\subsubsection{Acupuncture usage in primary care}

Most respondents $(n=16)$ reported some demand for acupuncture, mainly quarterly or less frequent enquiries $(n=9)$. Six GPs reported providing no access despite receiving enquiries. The two GPs who practised acupuncture were also among the minority of three respondents reporting a higher rate of solicitation than quarterly. The most frequently cited reason for which patients requested acupuncture was pain management. However, several other conditions were also mentioned, as represented in Fig. 2.

Barriers to acupuncture provision, in addition to a direct multiple choice question, emerged as one of the two themes identified by qualitative analysis of the open comments (see Box 1). The GPs who did not provide access to acupuncture cited the following reasons for this choice: (a) availability/difficulty in finding a trustworthy practitioner $(n=4)$; (b) cost and lack of funding $(n=3)$; (c) lack of patient demand $(n=3)$; (d) lack of knowledge $(n=3)$; (e) CCG controlling access $(n=1)$ and a general comment "not easily available", which may point to any or all of the logistical problems mentioned above. The claim about lack of patient demand contrasted with the fact that several respondents $(n=6)$ did receive patient enquiries but still provided no access. Only one respondent (trained in the USA) specified his negative view of CAM as the reason for providing no access.

\subsubsection{General practitioners' attitude to acupuncture}

Attitudes reflected in multiple choice questions, 1-10 scales and optional open comments ranged from considerable enthusiasm $(n=2)$ to strong objections $(n=1)$. Most respondents presented a moderate attitude, with two relatively sceptical and several, whether providers or non-providers, moderately favourable.

When rating acupuncture's benefit on a 1-10 scale (asked of respondents who provided acupuncture access through their practice, $n=8$ ), two assigned the maximum value of 10 ("very high benefit"), the remaining 6 respondents chose values between 5 and 8. Also, two respondents who did not provide acupuncture treatment nor referral answered the question, rating the benefit at 3; a third non-provider marked acupuncture as "potentially beneficial" without a numeral rating.

All respondents were also requested to assess the risk of acupuncture treatment on a 1-10 scale, where 1 represented very low, and 10 very high risk. The median value was $2(n=8)$. A binomial test was done (in statistical software "R") to check whether the distribution of higher versus lower risk responses 

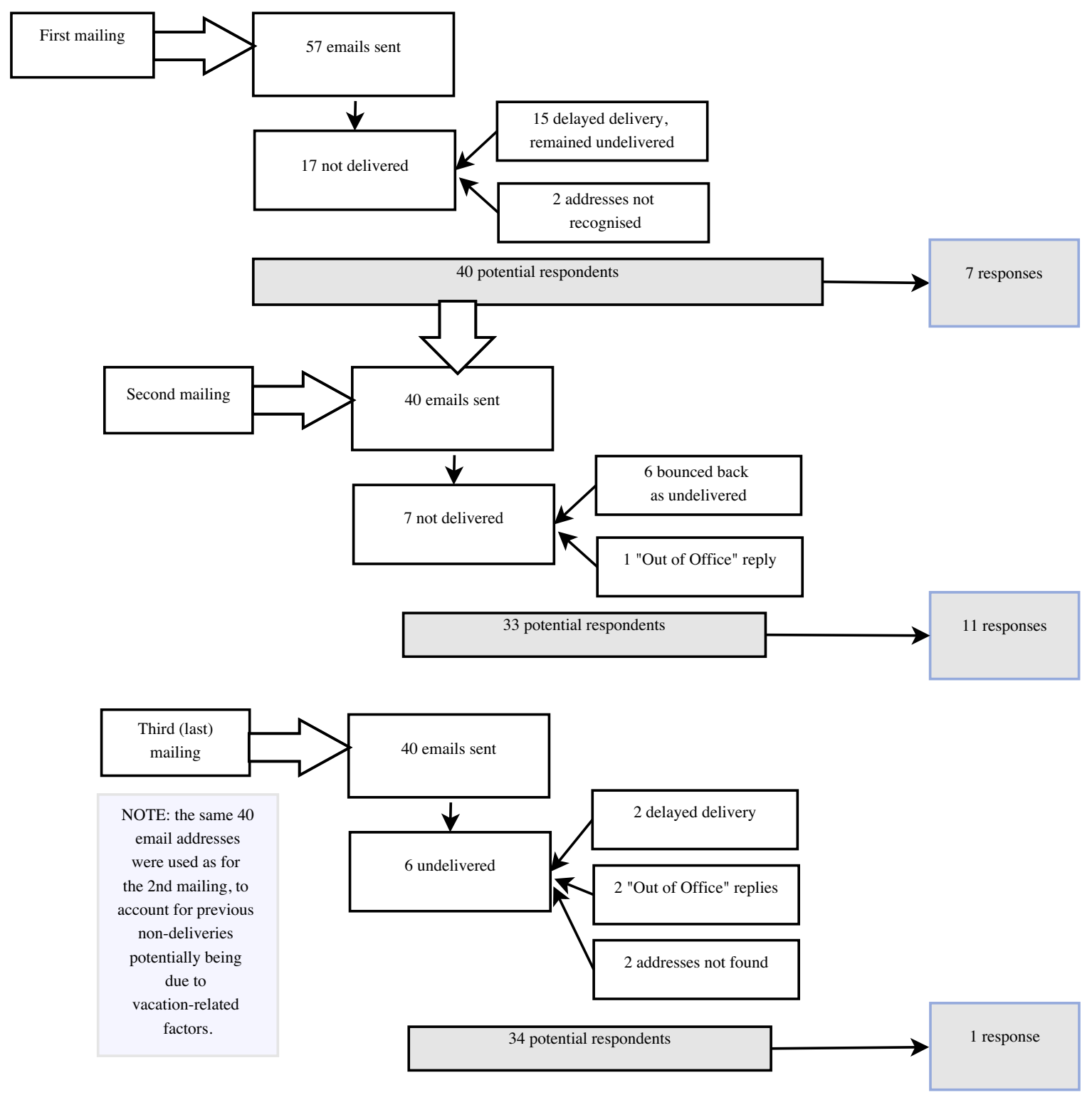

Fig. 1. Planned and actual sample size and response rate.

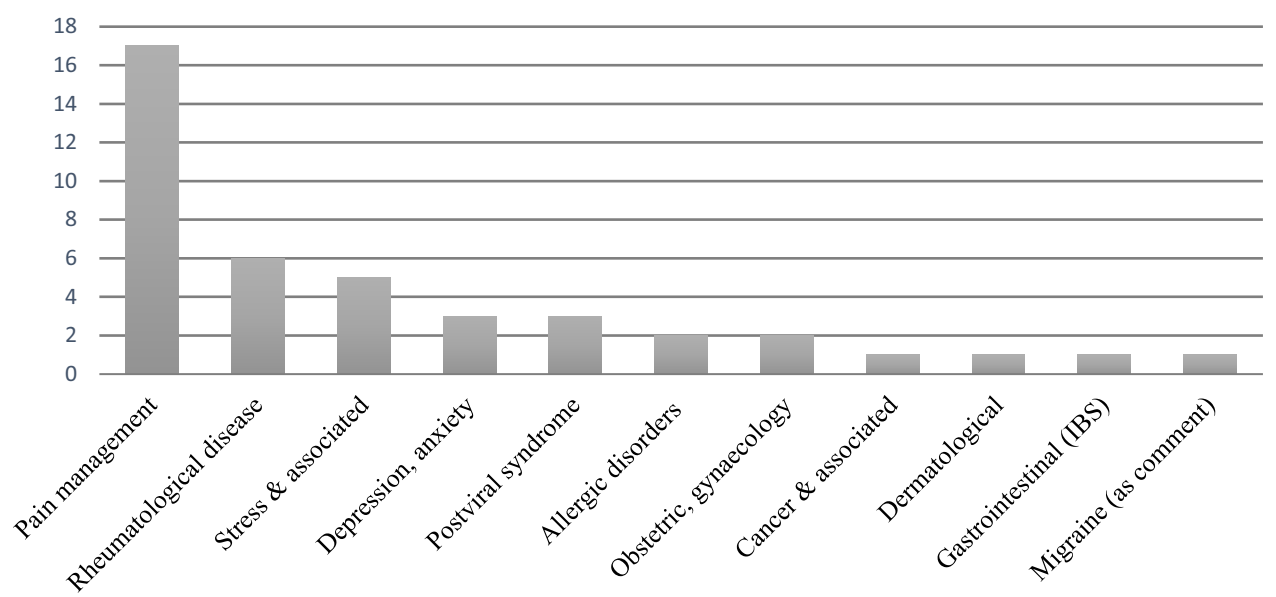

Fig. 2. Acupuncture enquiries by condition.

Please cite this article in press as: I. Czarnawska-Iliev, N. Robinson, General Practitioners' use of and attitudes to acupuncture in relation to the UK's National Institute for Health and Care Excellence (NICE) clinical guidelines-A pilot study, Eur. J. Integr. Med. (2016), http://dx.doi.org/ 10.1016/j.eujim.2016.07.004 
Box 1. Examples of open statements.

Theme 1: Barriers to provision

Access to acupuncture treatment is in the hands of health commissioners.

Unclear referral pathways and patchy provision under NHS.

We don't have anyone doing it.

Cannot be offered as a part of GP contract.

It would not be cost effective.

Patients mostly go through physios locally.

Theme 2: Attitude to acupuncture ${ }^{\mathrm{a}}$

Acupuncture works, NICE is not so reliable.

There is good evidence for its use in specific conditions, especially pain and nausea, and evidence of a very small risk of harm, e.g. pneumothorax. I have had acupuncture myself and am happy to refer for acupuncture. ${ }^{\text {b }}$

There is not only a lack of evidence, but there is some evidence of harm [and providing acupuncture] undermines my status as an allopathic practitioner and strengthens patient belief in alternative medicine.

a in addition to those open comments, three respondents chose "I don't know enough about it" in multiple choice questions.

${ }^{b}$ this respondent did not provide neither treatment nor referrals

could be attributed to something other than random incidence. The result was that to a $95 \mathrm{CI}$ the distribution was not random.

Respondents were also asked whether they would like to see NICE consider additional recommendations for acupuncture. A similar number of respondents chose "Yes" $(n=5)$ and "No" $(n=4)$, while most opted for a "Don't Know" and two skipped the question. Out of the 5 who chose "Yes", one provided no access through his practice. Among the conditions for which respondents wanted to see recommendations, various kinds of pain were most frequently mentioned: chronic, arthritic, knee, shoulder, musculoskeletal and cancer pain. There was also one mention each of stress and psychiatric conditions.

Spontaneous expressions of attitude towards acupuncture emerged as the second of the two themes reflected in the open comments. One reflected a strong negative perception of this treatment modality, and two considerable enthusiasm (see Box 1). One respondent who emphasised his positive attitude to acupuncture provided no access. Direct scepticism was not apparent multiple choice questions, as no respondent chose "I don't believe in it", or "I would be unlikely to use acupuncture anyway" in questions Q8 and Q12 respectively.

3.2.4. General practitioners' awareness of and attitude to guidelines

When asked to indicate for which conditions NICE recommended acupuncture, only one respondent provided correct answers for all the 18 illness categories, while four had zero correct - chiefly due to a high number of "I don't know" answers.
The mean number of correct answers was 6.15. The mean number of incorrect answers was 3.

General Practitioners tended to overestimate the incidence of NICE recommending either acupuncture treatment or its discussion. For each of the listed conditions, at least one respondent thought that NICE recommended either the discussion or use of acupuncture (Fig. 3). Given the high incidence of such claims for five categories, the full text of NICE guidance covering these conditions was consulted, but no additional recommendations were found.

The differences between the numbers of correct answers to different conditions were examined in SPSS to check for significant non-random distribution. As it is expected of that a GP should know about NICE guidelines, the results were treated in a binomial fashion of correct versus non-correct. A Cochran' $\mathrm{Q}$ test, determined that there was a statistically significant difference in the rate at which GPs mis-identified guidelines, $\mathrm{p}<0.0005$.

The question: "If NICE recommended acupuncture for a particular condition, would it make you more likely to use it for yourself?" was a marker of the importance GPs assign to guidelines for acupuncture. The heterogeneity of responses mirrored that in guideline recognition. The numbers of respondents who chose "much more likely" were very closely matched by "not more likely at all - I would take the decision based on research evidence", at 5 and 4 respondents respectively. Two answered "I don't know", which also implies poor trust in the guidance, betraying a disinclination to follow it per se. The majority, albeit by an

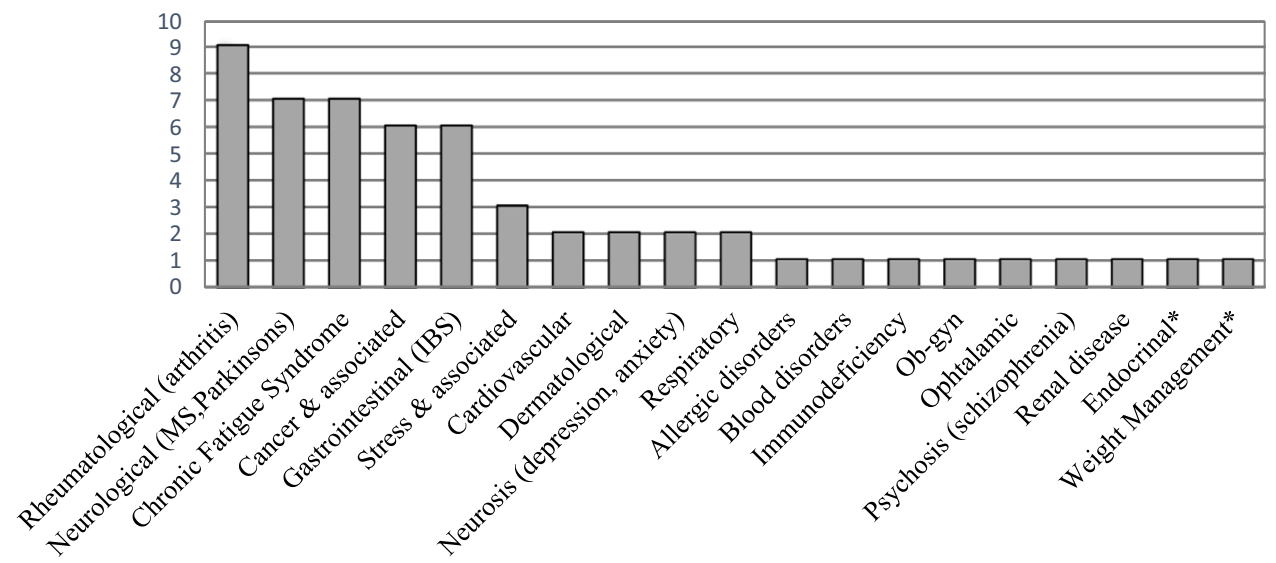

Fig. 3. NICE recommendation overestimates by condition. Asterix marks answers left in comment boxes. 
insignificant margin, chose "Somewhat more likely" $(n=6)$, while the remainder skipped the question. On the basis of this, respondents were subdivided into three groups: high trust in guidance, low trust in guidance and a relatively neutral majority. A descriptive analysis of the two extreme groups (Table 3 ) revealed that all but one from the "high trust" group did not provide acupuncture access, while all but one from the "low trust" group provided it. Also, both respondents who practised acupuncture were included in the latter group. Heterogeneity of correct guideline recognition within both groups was considerable.

The apparent inverse relationship between self-proclaimed trust in guidelines and acupuncture provision was explored in SPSS using Pearson's Chi Squared test. The test yielded a result of 0.048 , which is significant at $\mathrm{CI} 0.5$, however, at the same time the assumptions of the test were not met due to small sample size leading to low expected values. For a Pearson's Chi Square test to be valid, the expected value count has to be over 5 in at least $80 \%$ of cells [23]. The test does, however, hint at a correlation, due to a big difference in actual and expected values, reflected in the values of their respective adjusted residuals. Agrasti [24] elucidates their interpretation. If the distribution was random, the actual values would be close to the expected ones; the fact that they are not suggests that the distribution is not random, but this difference, when observed on the current sample size has no statistical meaning. At the same time, the test provides a basis for increased interest in the topic, which is reflected in the discussion.

\section{Discussion}

\subsection{Feasibility of future studies}

The role of a pilot study is to clear the path for future studies on a larger research sample. This study helped to identify challenges to feasibility as well as the strengths of research through an electronic questionnaire in general practice, and allowed to finetune the questionnaire for future usage.

A serious and unexpected challenge to feasibility was the number of incorrect email addresses. We have identified three ways in which future studies may attempt to deal with this problem:
- Overestimating sample size by about 60\%: In this study, the number of undelivered emails (23) constituted $67 \%$ of the remaining sample (34). Thus, assuming a consistent ratio of error in the CCGs databases, to sample 100 GPs, attempting to contact 180 email addresses would be necessary. This method, while logistically the simplest, precludes sampling the whole population.

- Sourcing email addresses directly from surgeries: this is much more labour-intensive, for which reason it may be unfeasible on a national scale without funding. Inaccuracies may still be present, however, it seems the preferable way to carry out a small or medium scale study. Practice managers could be contacted to request discussing the research at the weekly practice meeting, soliciting a list of emails of the current GP staff. Sufficient time for such solicitations must be allowed for in the study's timeframe.

- A postal questionnaire mailed to surgeries: a questionnaire would be undelivered only if a GP ceased to work at a particular surgery or if it closed. However, the success of returning completed questionnaires is unknown and there could be some bias operating regarding who completed the paperwork.

Moreover, according to SurveyMonkey's in-built response timer, some respondents spent roughly half of the predicted time of $10 \mathrm{~min}$ answering the survey; the answers of those respondents were imprecise, for example answering questions they were told to skip or failing to detail their answers when choosing "other". Given the GPs' work load, a rushed style of answering the questionnaire should be taken into account in future studies by making the questionnaire more precise (see below). Also, following up with telephone interviews in order to clarify answers where needed would be beneficial. An attempt was made during this study to see whether GPs would be open to such further telephone contact by piloting an additional question to half of the respondents and following up with an email interview invitation where appropriate. This demonstrated that in practice this is very difficult to do and, as discussed below, carries a substantial risk of bias. Future researchers should think very carefully about interviewing recruitment and method should they wish to interview GPs over the phone.

Table 3

Importance assigned to guidelines versus acupuncture provision.

\begin{tabular}{|c|c|c|c|c|c|}
\hline \multicolumn{6}{|c|}{ "High trust" group profile } \\
\hline \multirow[t]{2}{*}{ respondent code } & \multirow[t]{2}{*}{ acupuncture access $(\mathrm{Y} / \mathrm{N})^{\mathrm{a}}$} & \multirow[t]{2}{*}{ guidance trust $(0-2)^{b}$} & \multicolumn{3}{|c|}{ guideline recognition } \\
\hline & & & correct & incorrect & "don't know" \\
\hline R3 & $\mathrm{N}$ & 2 & 15 & 1 & 2 \\
\hline R5 & $\mathrm{N}$ & 2 & 3 & 15 & 0 \\
\hline R13 & $\mathrm{N}$ & 2 & 11 & 3 & 4 \\
\hline R16 & $\mathrm{N}$ & 2 & 7 & 2 & 9 \\
\hline $\mathrm{R} 18$ & $\mathrm{Y}$ & 2 & 16 & 2 & 0 \\
\hline Mean values: & & & 10.4 & 4.6 & 3 \\
\hline \multirow[t]{2}{*}{$\begin{array}{l}\text { "Low trust" group profile. } \\
\text { respondent code }\end{array}$} & acupuncture access $(\mathrm{Y} / \mathrm{N})^{\mathrm{a}}$ & guidance trust $(0-2)^{\mathrm{b}}$ & \multicolumn{3}{|c|}{ guideline recognition } \\
\hline & & & correct & incorrect & “don't know" \\
\hline R2 & Y (self) & 0 & 0 & 0 & 18 \\
\hline R9 & $\mathrm{Y}$ & 0 & 1 & 2 & 15 \\
\hline R15 & $\mathrm{Y}($ self $)$ & 0 & 18 & 0 & 0 \\
\hline R17 & $\mathrm{N}$ & 0 & 13 & 5 & 0 \\
\hline R12 & $\mathrm{Y}$ & “don't know” & 3 & 1 & 14 \\
\hline R19 & $\mathrm{Y}$ & "don't know" & 0 & 2 & 16 \\
\hline Mean values: & & & 5.83 & 1.67 & 10.5 \\
\hline
\end{tabular}

a $\mathrm{N}=$ provides no access, $\mathrm{Y}=$ refers for acupuncture, $\mathrm{Y}$ (self) = offers treatment, possibly in addition to referrals.

b "guidance trust" is based on Q12:2 = "much more likely" to use acupuncture for self if recommended by NICE; $0=$ "not more likely at all". 
Altogether, it appears that gathering data from GPs through electronic questionnaires holds promise. Sufficient data was gathered to allow insights into all the study objectives. Respondents answered even long multiple-choice questions thoroughly. About half $(n=10)$ left optional open comments and these reflected both positive and negative attitudes to acupuncture. There was a surprisingly high degree of willingness to be contacted by researchers (8/16 respondents), which can be seen as a marker of those respondents' willingness to help in research. However, the group that agreed to contact included over twice the number of respondents providing acupuncture access as compared to the group not agreeing to further contact (5 vs 2 ). That fact, as well as the heterogeneity of the responses proves that interviews or focus groups, which limit the number of participants compared to questionnaires, run a high risk of examining only a part of the picture.

Insights from this study can be used to improve the questionnaire for future use. Firstly, it should allow for greater complexity of GPs' views by letting respondents not offering acupuncture access to discuss its benefits. Secondly, in multiple choice questions, the options of "other" should always automatically require details. Thirdly, moving the demographic section to the beginning of the survey should be considered, as some respondents quit just before providing this information.

\subsection{Part 1: acupuncture in NICE guidelines}

The results of our updated guidance search points to the fact that in the majority of cases (29 out of 35), acupuncture or acupressure evidence is not considered. For acupuncture to be included as a potential recommendation in a NICE guideline, in the first place, relevant evidence must be sought by the Guidance Development Group (GDG), secondly, such evidence must be available and of high quality, and thirdly, it must be correctly interpreted. Therefore, not seeking such evidence precludes possible integration at the very beginning of the guidance development process. Incidentally, even in China, where acupuncture is widely practised alongside Western medicine, those two medical systems are viewed as separate to such a degree that only a minority of Western clinical guidance mentions it [25]. Also, the interpretation of the available evidence may be another stumbling block to integration: while GDG are multi-disciplinary committees, they may not include an acupuncture or CAM practitioner or researcher. Because of the vastly different paradigms underlying Western and Eastern medical systems, such a person would be better positioned to ask the correct questions of the available evidence.

On the other hand, the four guidelines identified in this study which discussed acupuncture evidence without making a recommendation, fulfil an important role by helping to identify gaps in research. As Lorenc et al. observed [1], the relationship between guidance and research is a dynamic one: clinical guidelines may fail to account for emerging evidence, while pointing to lacking evidence. Interestingly, even CG171: Urinary Incontinence, while making a "do not use" recommendation for acupuncture, states that: "Poor-quality evidence shows that acupuncture may reduce nocturia and both stress and urgency incontinence in the short term" (emphasis added). The availability of scientifically robust studies is a pressing issue. Access to literature on acupuncture may be limited to a GDG, as many studies are available in Chinese language only. Simultaneously, Chinese research often lacks robustness by Western standards, which is poignantly explained by MacPherson et al. as stemming from the difference in questions the researchers seek to answer [26]. Given the holistic and personal nature of Traditional Eastern Medicines, research that meets rigorous Western standards is difficult to design. Therefore, for integration to be possible, "It is vitally important that competent professionals, both clinically skilled and trained in research are involved in the development of the evidence base to ensure that it is appropriate and ongoing" [27].

\subsection{Part 2: insights from the questionnaire}

Given the small research sample inherent to a pilot study, the insights discussed below should be seen as signposts for exploration in further research rather than conclusions in their own right.

The first research objective of this study was to assess acupuncture usage in primary care. Patient demand appeared wide-spread but small: roughly $3 / 4$ of respondents reported receiving enquiries, but mostly no more frequently than quarterly. Although questionnaire data is subject to recall bias, as a GP sees on the average 30-40 patients a day [28], the findings still suggest that acupuncture is only discussed at a fraction of a percentage of the consultations. This echoes the results of a 1995 national survey of access to CAM via General Practitioners [29], which found access to be "widespread", but "to affect a relatively small number of patients." Therefore, quite possibly, not much has changed in the access to acupuncture through General Practice in the last 20 years, despite several years of NICE guidelines endorsement of acupuncture for certain conditions and a rising body of evidence. Further research is needed to support this suspicion.

Also, the reasons for acupuncture being discussed so infrequently during GP consultations may need to be explored. It is possible that patients are unaware of possible access to this treatment modality on the NHS, afraid to bring it up, or do not think of it when visiting a GP. Also, GPs who are positively inclined towards acupuncture may fail to discuss it because of timeconstraints, or, as is suggested by a pilot of an electronic template to discuss CAM for eczema [30], "due to busy, target-driven practice". Finally, both demand and provision might be influenced by Brent's high ethnic and economic diversity, as the use of CAM varies among ethnic minorities. Studies in the UK and USA demonstrated that acupuncture is more popular among (nonHispanic) white population [31-33], while a previous study of almost the same population as this pilot (Brent and Harrow) found South Asians to be much less likely to use it, favouring instead herbs and Ayurveda [31]. While that study is rather old, such preferences are deeply rooted in the cultural outlook and would be slow to change. Additionally, a British national survey found a significant association between the use of CAM and non-manual social class, as well as better education and higher income [34]. Results of a similar study in a homogeneous ethnic and social environment might be rather different.

Our results also suggest a gap between acupuncture demand and provision, as at least 6 out of the 16 GPs who received enquires offered no treatments nor referrals. This affects mostly the less economically privileged patients, for whom payment for private treatment constitutes a barrier. Therefore, this gap emerges as an important niche to explore in further research, and makes understanding the reasons behind GPs providing access or refraining from it all the more pertinent. It is possible, on the one hand, that a number of patients' acupuncture enquiries are medically unjustified or would not be cost effective. On the other hand, doctors in this study reported logistical barriers to provision, such as difficulties in finding a practitioner and lack of funding.

The second research objective was to explore GPs' attitudes towards acupuncture. The questionnaire captured a wide range of opinions, from considerable dislike to professional enthusiasm. It is interesting that the one respondent who openly expressed his negative view of acupuncture had received his medical training in the USA. While possibly a coincidence, it may be that due to 
cultural differences, he expressed his opinions more strongly. Attitudes are not always transparent, as evidenced by the fact that even that respondent refrained from choosing the openly sceptical answers in multiple choice questions. It is possible that the questionnaire results underestimate sceptical views, as GPs may refrain from expressing them.

Most respondents presented a moderately open attitude, as evidenced by a relatively high benefit rating and relatively low risk rating. An interesting result was that even some of the GPs not offering access $(n=3)$ expressed an opinion that acupuncture is potentially beneficial. It suggests that acupuncture attitude is not the main reason, but at most one of the variables, in deciding whether to offer access or not. Half of all respondents expressed a desire to see NICE consider recommending acupuncture for more conditions, while half did not - this may be seen as a marker of a favourable attitude towards this modality, though it could also be argued that they were being pragmatic and keen to see evidencebased recommendations. In this light, the fact that half did not think NICE should consider acupuncture more can be seen as a sign of scepticism towards either acupuncture or NICE.

The third research objective yielded potentially surprising insights into respondents' awareness of and attitude to official NICE guidelines for acupuncture. None of our findings revealed a direct link between NICE recommendations and acupuncture provision. Awareness of recommendation for pain management which appeared high among the respondents - did not translate in this study into referrals or treatment for pain. However, pain management for various conditions not currently included in NICE recommendations strongly emerged at the forefront of both patient demand and GP suggestions for further consideration by NICE. This is unsurprising, since much of the discourse on acupuncture in the West has focused on pain conditions [35], and other researchers report a high prevalence of acupuncture provision for pain, followed by nervous diseases [31], neurological and psychological conditions [9]. Today, there appears to be good evidence for the efficacy of acupuncture for several musculoskeletal conditions, such as neck or shoulder pain [5], which is currently not reflected in NICE guidelines.

Another finding which suggests a degree of disjuncture between NICE recommendations and clinical practice is that respondents appeared to expect recommendations where there were none, and the rate of overestimating NICE recommendations was not statistically uniform. It may imply that the respondents thought that acupuncture would be useful for the conditions for which they overestimated recommendations the most, such as psychological and neurological conditions, especially that for those same conditions doctors are reported to provide treatments most often [9]. Interestingly, several GPs reported demand, and some assumed a NICE recommendation, for conditions for which NICE lists acupuncture as a "do not use" intervention, chiefly gastrointestinal problems such as IBS, and arthritis. Admittedly, there is a degree of controversy surrounding NICE arthritis anti-recommendation: the decision not to recommend acupuncture for osteoarthritis was reached on economic grounds, but Latimer et al. [36] found inconsistencies with regards to using different comparator groups for different ailments, and demonstrated an economic advantage in their re-analysis. Also, systematic reviews of IBS trials report significant, though possibly non-specific, benefits of acupuncture treatment $[37,38]$. Therefore, clinical reality appears to reflect pragmatic evidence to a larger degree than it does NICE guidance

An element which undoubtedly contributes to many GPs' relatively poor knowledge of the guidelines is the oft-quoted time pressure, which makes familiarisation with guidelines difficult $[7,8]$, especially given the considerable volume of recommendations and guidelines [8]. This aspect may be getting steadily worse, as new guidance is published while the pressures on primary care are increasing, especially in Brent [20].

Lastly, a surprising finding of the study was a tendency of scepticism towards NICE guidelines correlating positively with providing acupuncture access. This relationship was strong enough to be detectable statistically even on our small research sample. One way to illuminate this correlation is through the prism of research on mindsets. The underlying premise of the theories developed by Zerubavel [39] and more recently Dweck [40,41] is that some individuals perceive the world as organised along stable, fixed categories, while to others boundaries seem "fuzzy", and reality in a state of constant flux. Individuals prone to following an official guideline per se are likely to belong to the first category of a "fixed" mindset, and therefore also perceive a deeper and stricter division between the realm of primary care and CAM. On the other hand, individuals with a more "flexible" or "fuzzy" mindset might be both disinclined to follow an authority and perceive a blurring of the lines between biomedicine and CAM. Another explanation for this tendency might be that individuals inclined to offer acupuncture access perceive NICE as reflective of a medicalised or Westernised approach (congruent with one respondent's comment: "NICE is not so reliable"). However, qualitative research is needed to test this more in depth and indeed to prove whether such a correlation remains on a larger scale.

\subsection{Limitations of the study}

The chief limitation of this study is its modest participation rate, engendering non-response bias. GPs might be more likely to partake if they had strong opinions about acupuncture, or wanted to promote its integration with biomedicine. Therefore, GPs enthusiastic about acupuncture may be over-represented. Response rate could have been improved by minimising the number of incorrect email addresses within the sample ( $40 \%$ in this study), as discussed under "Feasibility", as well as by conducting research outside of the holiday period. Also, the reasons why some respondents quit the questionnaire early should be considered. As no respondents quit around the most personal question (Q12), they may have simply forgot or been discouraged by the number of electronic "pages". It would be useful to compare response rates to electronic questionnaires formatted into more but shorter, or fewer but longer pages. At the time of writing, no such studies have been identified.

Additionally, we have identified two potential limitations of this study's method of assessing guideline awareness. Firstly, since most responses are "no", respondents generally sceptical of acupuncture may have a similar score as those with an interest in and knowledge of guidelines for acupuncture. Secondly, guideline awareness was not assessed along the same categories as used in the guidelines for acupuncture. For instance, the questionnaire did not ask about low back pain and irritable bowel syndrome, but more generally about pain management and gastrointestinal/digestive conditions. The decision to phrase questions this way was in order to avoid phrasing which may be leading, but rather take clinical experience as the starting point. Given the high incidence of responses regarding various categories of pain, it could have been better instead to provide subcategories of pain as separate answer options.

It should also be pointed out that in this study, acupuncture was viewed in a simplified way as one modality, when in reality it is a heterogeneous and multi-faceted family of therapies [26,35]. While a Western model of medical acupuncture is most popular among doctors (and indeed the information gathered about acupuncture training of respondents is consistent with that assumption), it is possible that referrals involved a variety of 
acupuncture styles, seen by their respective practitioners as quite disjointed.

Lastly, the study did not examine respondents' personal and familial use of acupuncture, which would have provided a fuller picture of their individual normative beliefs. Previous studies suggest a tension between the private and professional realms for medical professionals [42]. Respondents had opportunities to mention their personal experience if they considered it relevant. Only one respondent mentioned having had acupuncture.

\section{Conclusion}

Part 1 of this study updated the knowledge of acupuncture in NICE guidelines published until July 2015, finding one new “do not offer" recommendation, and attesting to the fact that this treatment modality is considered relatively rarely in the guidance development process. When it is considered, high quality evidence is deemed to be lacking. Acupuncture researchers might take into account the instances where the GDG did search for acupuncture evidence as a call for more high quality research in those areas.

Part 2 has demonstrated that, despite the time pressures in general practice, an electronic survey can be a useful tool of data collection. However, inconsistencies in email addresses pose a challenge and need to be carefully addressed in future studies.

In addition, the questionnaire survey results pointed to several topics worthy of further exploration. They suggests a gap in acupuncture demand and provision in primary care. While some GPs successfully include acupuncture in their practice, others do not provide treatment nor referral, even when they appear to be positively inclined towards this treatment modality. Lack of knowledge, lack of funding, difficulties in finding a practitioner, unclear referral pathways and control by the CCG where among the reasons cited for lack of provision. Qualitative interview-based studies could explore in detail the reasons behind why some GPs do not offer acupuncture access despite professing a positive attitude towards it, and GPs' views on integration. Other questionnaire-based studies could be designed to explore why acupuncture is being discussed so infrequently during GP consultations.

Meanwhile, acupuncture professional organisations, such as the British Acupuncture Council, could endeavour to decrease the knowledge gap by sending information leaflets to surgeries, or offer practice managers a visit at their weekly meeting, and work with NHS bodies to design appropriate Continuing Professional Development courses.

Repeating this study, while incorporating changes discussed under "feasibility", on a larger scale, would allow to test conclusions suggested by our results regarding GPs awareness and expectation of guidance for acupuncture. Our respondents appeared mostly aware of NICE recommending acupuncture as a treatment option for pain, but many also ascribed such a recommendation to several other conditions, including those where NICE expressed a "do not use" guidance (IBS, arthritis). In this study, these overestimates appeared to reflected patient demand. NICE should be aware that many patients and GPs have a favourable perception of acupuncture use for some of the conditions for which there is currently a "do not use" recommendation, chiefly arthritis and IBS, as well as for several musculoskeletal, neurological, and psychological conditions. Those guidelines appear worth revisiting as evidence body grows.

The findings of this study come at a time when the most longstanding NICE recommendation for CAM (acupuncture) is about to be removed from the revised guideline on low back pain. They suggest that this revision is unlikely to change acupuncture access through primary care, as awareness of NICE guidance on acupuncture did not appear to influence provision within our sample. It might of course have indirect effects, which are harder to quantify.

Conversely, a sceptical attitude towards NICE guidance correlated positively with offering acupuncture access. A suggested link between mindset and CAM integration into general practice could be explored through focus groups. An understanding of this topic is important not only to allow patients access to a range of effective treatment modalities, but also as a potential avenue to help general practitioners cope with an aging patient population and local pressures on primary care [20], such as are currently faced by the surgeries in Brent.

\section{Financial support}

None.

\section{Conflicts of interest}

None.

\section{Acknowledgements}

The study was conducted as a part of a Masters Program at London South Bank University. The authors would like to thank Dr. Banarsee for facilitating contact with General Practitioners in the Brent CCG and providing a random sample of respondents.

\section{Appendix A. Questionnaire}

Investigating General Practitioners' use of and attitudes to acupuncture in relation to current National Institute of Health and Care Excellence (NICE) clinical guidelines

1. In the last 12 months, how often have your patients asked for acupuncture?

Never

Once or twice

Quarterly

Monthly

Weekly

Daily

Other: (give details) (comment box)

2. For what kind of conditions do patients request acupuncture, if they do?

Drop down list of conditions (as in Q10)

Other:_ (comment box)

3. Do you provide any acupuncture in your practice?

No

Yes - myself

Yes - one of the other GPs

Yes - someone else in the practice. Please give details (e.g. independent acupuncturist, physiotherapist, practice nurse etc.) (comment box)

4. Do you refer patients for acupuncture outside of your practice?

yes/no

5 . If you do refer for or provide acupuncture, how often have you done so in the last 12 months?

Once or twice

Quarterly

Monthly

Weekly

Daily

Other: (give details) (comment box)

6. If you do refer for or provide acupuncture, is this because you think it is (tick all that apply):

Beneficial 
Potentially beneficial

Something that patients request

Accepted by commissioners

Successfully used by colleagues

Other (specify):

(comment box)

7. If you chose "Beneficial" or "potentially beneficial", please indicate on the 1-10 scale below how would you rate the health benefit of acupuncture therapy.

(SCALE)

8. If you do not either offer acupuncture in your practice or refer to an acupuncturist outside of your practice, why is this? (tick all that apply)

Patients have not asked

I do not believe in it

There is insufficient evidence

It would not be cost effective

Cannot be offered as part of the GP contract

Do not know how to find an appropriate practitioner

Potential safety issues

Not easily available

Lack of statutory regulation

I don't know enough about it

Other (specify): (comment box)

9. Please indicate on the 1-10 scale below, what, in your opinion, is the level of risk associated with acupuncture treatment.

(SCALE)

Or: I don't know

10. Do you know whether NICE recommends the use of acupuncture for any of the following conditions:

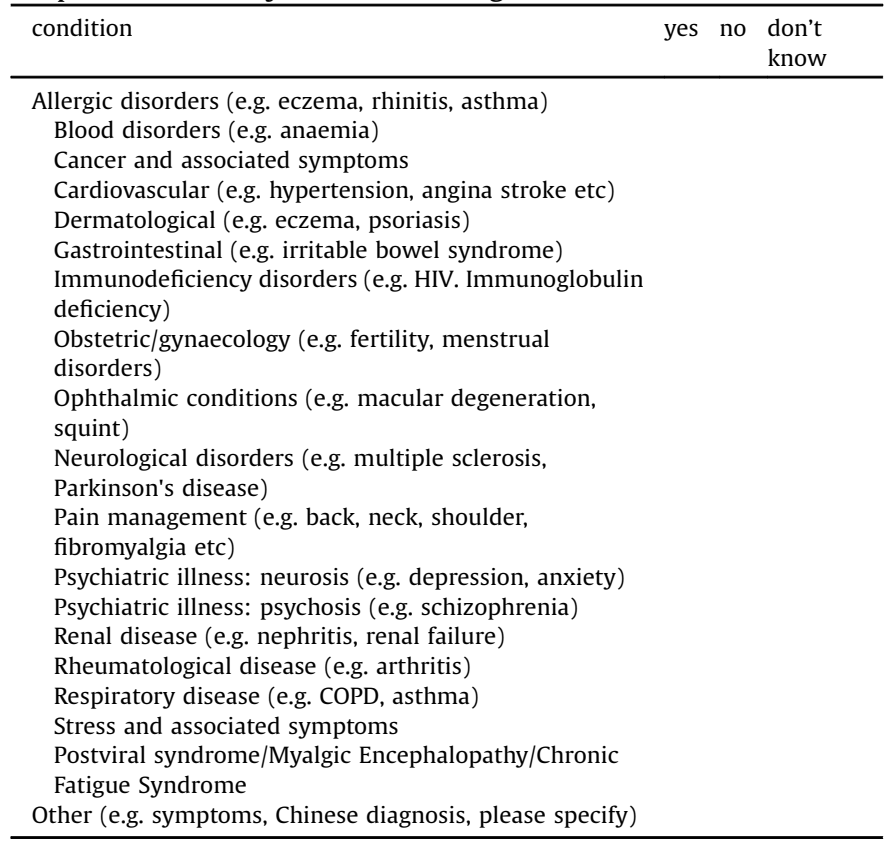

11. Do you think NICE should consider recommending the use of acupuncture for any other conditions?

yes

no

don't know

If yes, for what condition(s):

(a drop down list of conditions as in Q10 and comment box for "any other")

12. If NICE recommended acupuncture for a particular condition, would it make you more likely to use it for yourself?
Not more likely at all - I would be unlikely to use acupuncture anyway

Not more likely - I would take the decision based on research evidence

Somewhat more likely

Much more likely

Don't know

13. (Optional - a clearly visible SKIP option in the interface)

If you would like to share any thoughts about acupuncture or NICE guidance for acupuncture, please do so here:

(Comment box)

Personal Data

Are you

male/female

Age range:

- under 29

- 30-39,

$-40-49$

$-50-59$,

$-60+$ box)

Country you trained in - (drop down list of countries + "other"

Do you have any training in acupuncture? Yes/No

If yes, what training and where:

(comment box)

Your experience and opinions are very valuable. Can the researchers contact you should they need to clarify something? Yes/No.

If yes, please provide your email address below.

If you would like to be contacted with the results of this study, please email: czarnawi@lsbu.ac.uk or nicky.robinson@lsbu.ac.uk.

\section{References}

[1] A. Lorenc, J. Leach, N. Robinson, Clinical guidelines in the UK: do they mention complementary and alternative medicine (CAM) - are CAM professional bodies aware? Eur. J. Integr. Med. 6 (2014) 164-175.

[2] K. Trinh, N. Graham, A. Gross, C.H. Goldsmith, E. Wang I.D. Cameron, T. Kay, C. O. Group, Acupuncture for neck disorders, Cochrane Database Syst. Rev. 3 (2006).

[3] L.-M. Fu, J.-T. Li, W.-S. Wu, Randomized controlled trials of acupuncture for neck pain: systematic review and meta-analysis, J. Altern. Complement. Med. 15 (2009) 133-145.

[4] J. Vas, J.M. Aranda-Regules, M. Modesto, M. Ramos-Monserrat, M. Barón, I. Aguilar, N. Benítez-Parejo, C. Ramírez-Carmona, F. Rivas-Ruiz, Using moxibustion in primary healthcare to correct non-vertex presentation: a multicentre randomised controlled trial, Acupunct. Med. 31 (2013) 31-38.

[5] A.J. Vickers, A.M. Cronin, A.C. Maschino, G. Lewith, H. MacPherson, N.E. Foster K.J. Sherman, C.M. Witt, K. Linde, A.T. Collaboration and others, Acupuncture for chronic pain: individual patient data meta-analysis, Arch. Int. Med. 172 (2012) 1444-1453.

[6] I. Genevaise-Monié, C. Rat, C. Mouglalis, L'acupuncture dans la prise en charge des nausées et vomissements gravidiques, Acupuncture et Moxibustion 8 (2009) 204-212.

[7] A. Rashidian, M.P. Eccles, I. Russell, Falling on stony ground? A qualitative study of implementation of clinical guidelines' prescribing recommendations in primary care, Health Policy 85 (2008) 148-161.

[8] C. Heneghan, R. Perera, D. Mant, P. Glasziou, Hypertension guideline recommendations in general practice: awareness, agreement, adoption, and adherence, Br. J. Gen. Pract. 57 (2007) 948-952.

[9] A. Hopton, S. Curnoe, M. Kanaan, H. MacPherson, Acupuncture in practice: mapping the providers, the patients and the settings in a national crosssectional survey, BMJ Open 2 (2012) e000456.

[10] J. Ratcliffe, K. Thomas, H. MacPherson, J. Brazier, A randomised controlled trial of acupuncture care for persistent low back pain: cost effectiveness analysis, Br. Med. J. 333 (2006) 626.

[11] S. Barclay, C. Todd, I. Finlay, G. Grande, P. Wyatt, Not another questionnaire! Maximizing the response rate, predicting non-response and assessing nonresponse bias in postal questionnaire studies of GPs, Fam. Pract.19(2002) 105111. 
G Mode

EUJIM 576 No. of Pages 13

I. Czarnawska-Iliev, N. Robinson/European Journal of Integrative Medicine $x x x$ (2016) $x x x-x x x$

13

[12] E. Cottrell, E. Roddy, T. Rathod, E. Thomas, M. Porcheret, N.E. Foster, Maximising response from GPs to questionnaire surveys: do length or incentives make a difference? BMC Med. Res. Methodol. 15 (2015) 1.

[13] T. Yamane, Statistics an Introductory Analysis, Ind ed., Harper and Row, New York, 1967.

[14] A.N. Oppenheim, Questionnaire Design, Interviewing and Attitude Measurement, Pinter Publishers, London, 1992.

[15] W.H. Faddy, Constructing Questions for Interviews and Questionnaires: Theory and Practice in Social Research, Cambridge University Press, Cambridge, 1994.

[16] D. Luff, K. Thomas, Sustaining complementary therapy provision in primary care: lessons from existing services, Complement. Ther. Med. 8 (2000) 173179.

[17] A. Lorene, M. Blair, N. Robinson, Parents' and practitioners' differing perspectives on traditional and complementary health approaches (TCAs) for children, Eur. J. Integr. Med. 2 (2010) 9-14.

[18] ONS. Office of National Statistics, The 2011 Census: a profile of Brent. https:// intelligence.brent.gov.uk/BrentDocuments/Brent\%202011\%20Census\% 20Profile.pdf, 2011.

[19] Brent Clinical Commissioning Group, Annual Report: 2014-15. brentccg.nhs. uk, 2015.

[20] Brent Scrutiny Committee, Access to Extended GP services and Primary Care in Brent: Interim Report. www.democracy.brent.gov.uk/, 2015.

[21] L. Spencer, J. Richie, W. O'Connor, G. Morrell, R. Ormston, Analysis in practice, in: J. Ritchie, J. Lewis, C. MacNaughton Nichols, R. Ormston (Eds.), Qualitative Research Practice: a Guide for Social Science Students and Researchers, Sage Publications, 2013, 2016, pp. 295-346.

[22] Centre for Workforce Intelligence, In-depth review of the general practitioner workforce: final report. http://www.cfwi.org.uk/publications, 2014.

[23] D. Yates, Practice of Statistics, W. H Freeman \& Company, New York, 1999.

[24] A. Agrasti, Categorical Data Analysis, Wiley, New York, 2002.

[25] J. Ken, X. Li, J. Sun, M. Han, G.-Y. Yang, W.-Y. Li, N. Robinson, G. Lewith, J.-P. Lu, Is traditional Chinese medicine recommended in Western medicine clinical practice guidelines in China? A systematic analysis, BMJ Open 5 (2015) e006572.

[26] H. MacPherson, R. Hammerschlag, G. Lewith, Acupuncture Research: Strategies for Establishing an Evidence Base, Churchill Livingstone, Edinburgh, 2007.

[27] N. Robinson, Should acupuncture play a greater role in healthcare? International Symposium on Acupuncture Research, Bologna, Italy, 2015.
[28] Medical Careers, General Practice: Working Life, NHS, 2015.

[29] K. Thomas, M. Fall, G. Parry, J. Nicholl, National Survey of Access to Complementary Health Care via General Practice: Final Report to the Department of Health, University of Sheffield, School of Health and Related Research, Sheffield, 1995, pp. 11-37.

[30] N. Robinson, A. Loren, A. Falinski, R. Banarsee, The challenges of facilitating primary healthcare discussions on traditional, complementary and alternative medicine for childhood eczema: piloting a computerized template, Patient Educ. Coins. 89 (2012) 517-524.

[31] A. Greenfell, N. Patel, N. Robinson, Complementary therapy: general Practitioners' referral and patients' use in an urban multiethnic area, Complement. Sher. Med. 6 (1998) 127-132.

[32] T. Xu, T.W. Farrell, The complementarity and substitution between unconventional and mainstream medicine among racial and ethnic groups in the United States, Health Servo. Res. 42 (2007) 811-826.

[33] L. Villa-Caballero, C.M. Morello, M.E. Chynoweth, A. Prieto-Rosinol, W.H. Polonsky, L.A. Palinkas, S.V. Edelman, Ethnic differences in complementary and alternative medicine use among patients with diabetes, Complement. Ther. Med. 18 (2010) 241-248.

[34] K. Thomas, P. Coleman, Use of complementary or alternative medicine in a general population in Great Britain: results from the National Omnibus survey, J. Public Health 26 (2004) 152-157.

[35] S. Birch, R. Felt, Understanding Acupuncture, Churchill Livingstone, Edinburgh, 1999.

[36] N.R. Latimer, A.C. Bhanu, D.G. Whitehurst, Inconsistencies in NICE guidance for acupuncture: reanalysis and discussion, Acupunct. Med. 30 (2012) 182-186.

[37] E. Manheimer, L.S. Wieland, K. Cheng, S.M. Li, X. Shen, B.M. Berman, L. Lao, Acupuncture for irritable bowel syndrome: systematic review and metaanalysis, Am. J. Gastroenterol. 107 (2012) 835-847.

[38] G.-Q. Char, S. Zhang, Effectiveness of acupuncture to treat irritable bowel syndrome: a meta-analysis, World J. Gastroenterol. 20 (2014) 1871.

[39] E. Zerubavel, The Fine Line: Making Distinctions in Everyday Life, University of Chicago Press, Chicago, 1993.

[40] C.S. Dweck, Self-Theories: Their Role in Motivation, Personality and Development, Psychology Press, Philadelphia, 1999.

[41] C.S. Dweck, Mindset: How You Can Fulfill Your Potential, Ballantine Books, New York, 2006.

[42] A. Lorene, M. Blair, N. Robinson, Personal and professional influences on practitioners' attitudes to traditional and complementary approaches to health in the UK, J. Tradit. Chin. Med. Sci. 1 (2015) 148-155.

Please cite this article in press as: I. Czarnawska-Iliev, N. Robinson, General Practitioners' use of and attitudes to acupuncture in relation to the UK's National Institute for Health and Care Excellence (NICE) clinical guidelines-A pilot study, Eur. J. Integr. Med. (2016), http://dx.doi.org/ 10.1016/j.eujim.2016.07.004 